\title{
Brief Analysis on Treatment of Apoplectic Hemiplegia through Combination of Massage and Modern Rehabilitation
}

\author{
Haikuan Wang, Liangbing Yang, Jihong Wang \\ Clinical Medical College of Acupuncture, Moxibustion and Rehabilitation, Guangzhou University of \\ Chinese Medicine, Guangzhou Guangdong, 510405, China
}

Keywords: Massage, Modern rehabilitation, Apoplectic hemiplegia, Treatment research.

\begin{abstract}
The acroparalysis left after stroke is the main factor affecting patients' activities of daily living, so limb rehabilitation training is particularly important for stroke patients. The modern western medical rehabilitation is dominated by active movement and patients are easy to have fatigue and inimical emotions during the training; however, the traditional Chinese medical massage, which is dominated by passive movement, can make up for the discomfort caused by active movement. Therefore, it is required to explore a more effective rehabilitation treatment method for apoplectic hemiplegia by organically combining traditional Chinese medical massage with the modern rehabilitation so that the hemiplegic patients can early recover their activities of daily living and then can return to the society early.
\end{abstract}

\section{Introduction}

After the acute phase is controlled, stroke patients are left with acroparalysis in varying degrees. Besides, arthralgia, stiffness, disuse amyotrophy and other symptoms are easily caused and will prolong the course of limb rehabilitation. As a part of China's traditional medicine, the science of Chinese massage has a long history and plays an active role in a hemiplegic patient's rehabilitation process. Besides, with its unique characteristics, modern rehabilitation method also has significant curative effect in promoting hemiplegic patients' functional recovery. With the increasing popularization of modern rehabilitation technology, treating apoplectic hemiplegia by combining massage with modern rehabilitation method becomes increasingly acceptable by patients. The combination of the two can complement each other's advantages and jointly facilitate the improvement of patients' condition.

\section{Dialectical combination of Brunnstrom stage and massage manipulation}

The rehabilitation of limb movement after stroke is actually the conversion process of motion pattern, combines the 6-stage theory for rehabilitation of movement function after stroke in modern rehabilitation science ${ }^{[1]}$, gives play to the advantages of treatment based on syndrome differentiation and holistic therapy in the science of Chinese massage, and carries out dialectical massage therapy according to patients' Brunnstorm stage. Brunnstrom proposed the 6-stage recovery theory. Firstly, the acute-stage affected limb is in the continuously slow state without any movement (Stage I); as the recovery begins, a patient has associated reaction, synergy movement, minimum voluntary movement reaction and spasm (Stage II); later, the voluntary emergence of synergy movement shows joint movements; thus the spasm is further aggregated and reaches the peak (Stage III); then, the synergy movement mode gradually abates; isolated movements appear; multiple movement combinations become easy; the spasm abates (Stage IV); A patient is further divorced from the synergy movement mode and can well complete independent movements and movement combinations with greater difficulties; the spasm continues abating (Stage V); finally, with the disappearance of spasm, a patient can finish each joint movement his/her coordination is almost normal (Stage VI). This theory conforms to a post-stroke hemiplegic patient's natural pathologic evolution process and recovery law, so it is required to adhere to the principle of painstakingly enhancing the muscular tension to shorten the flaccid paralysis period during the early rehabilitation of hemiplegia. According to the theory of 
traditional Chinese medicine, "Yangming is only effective to treat flaccidity syndrome", so we can treat a patient by using the rolling method to follow the lines on body surface along the Yangming meridians of hands and feet. As for the training method at this stage, Brunnstrom attains the goal through associated reaction or synergy movement and enhances the muscular tension on the affected side by forced shrinkage on the contralateral side. With the recovery of hemiplegia, the gradually increasing limb muscular tension will result in myospasml; at this time, the upper limb is mostly in the flexor spasticity while the lower limb is mostly in the extensor spasticity. During treatment, it is required to restrain the spasm; during massage therapy, it is required to avoid aggravating the spasticity of upper limb's flexor and lower limb's extensor. As flexor spasm easily appears in the upper limb ${ }^{[1]}$, when pinching the Deltoid muscle with five fingers, the doctor needs to ask the patient to painstakingly do the shoulder abduction movement; meanwhile, the doctor needs to give a certain resistance, ask the patient to stretch the elbow to the utmost, and ask the patient to stretch fingers to the utmost when pressing the Waiguan acupoint. Finally, the doctor places the patient's affected limb in the internally fixed position, asking the patient to painstakingly straighten the limb externally. The paralyzed lower limb is mostly in the extensor spasticity, is easy to recover standing and walking, but can easily form the circled pathological walking gesture. Therefore, when massaging the Huantiao point, the doctor should tell the patient to do internal rotation, adduction and inflection with the lower limb to the utmost and then kick out with at full tilt. At last, the doctor helps the passive flexion of lower limb at the hip point, knee bending, slight adduction and internal rotation on the affected side; then, let the patient to exert force from the waist to kick the limb at the utmost. If the patient fails to finish the movement well, the doctor can give assistance properly. A patient can form the correct walking ability through repetition of such massages and exercises. In short, with the growing muscular tension, massage therapy shall follow the principle of nervous physiology; otherwise, abnormal muscular tension cannot be restrained and the contrary effect may be caused.

\section{The evaluation of massage's curative effect needs to follow the theory of modern rehabilitation medicine}

The previous treatments of stroke used muscle strength and joint movement degree as the effective clinical assessment standards ${ }^{[2]}$ and unilaterally emphasized the recovery of muscle strength as the standard for effective massage therapy. However, as proved by practices, even though a stroke patient's muscle strength is enhanced or the strength of a single muscle group is very powerful through treatment, the patient's movement function of limbs on the affected side still cannot return to normal. Apoplectic hemiplegia is that the advanced nerve center losses its controllability for voluntary movement and thus the abnormal movement mode represented by myospasm under the control of lower center nerve is caused. Its essence is the qualitative change in the movement mode but not merely the change in muscle strength. As for hemiplegic patients, changes in the muscle strength cannot completely embody their disease changes. In particular, when a patient's muscle strength reaches Grade IV, the changes in muscle strength will be no longer obvious and the changes in muscular tension, walking gesture and other functions will occur. Then, if the doctor continues using the muscle strength as the observation index, not only it is very difficult for the doctor to figure out the curative effect but also joint deformity, ligament injury, spasm aggregation and other misuse syndromes will be caused due to the overemphasis on changes in muscle strength. Using the evaluation means of modern rehabilitation medicine, such as Ashworth scale (MAS), brief Fugl-Meyer assessment (FMA), improved Barthel index (BI) and special quality of life in stroke (SS-QOL), as development and beneficial supplements of the science of Chinese massage to evaluate the clinical effects of stroke patients at different stages from different perspectives can not only show patients' changes in dysfunction recovery before and after treatment but also can greatly help to guide patients' further treatment. 


\section{Massage can reduce the experience of technique application and enhance the scientificity by means of rehabilitation evaluation}

If the rehabilitation evaluation can be reasonably used in the process of treating hemiplegic patients through massage, the doctor can widely gather patients' subjective and objective data, compare, synthesize, analyze and interpret such data, and finally make correct judgments on the obstacle conditions. Therefore, the doctor can effectively improve the experience property of application of massage techniques and enhance the scientificity of technique application. For instance, using the evaluation of joint movement degree in a stroke-caused hemiplegic patient's massage treatment process has the following clear guidance significances for the application of massage techniques: firstly, determine the functional status of joint and select a reasonable massage technique. Through the evaluation of joint movement degree, the doctor can determine whether the joint movement is normal, find out the joint with abnormal movement, reflect the degree of abnormal joint movement with objective and correct numerical values, and thus figure out the functional status of joint so as to choose the reasonable massage techniques. Secondly, find out the causes for abnormal joint movement and use specific techniques accordingly. Nerve and muscle injuries etc. can result in abnormal joint movement. One can judge out the causes for a stroke-caused hemiplegic patient's abnormal joint movement by combining the evaluation of joint movement degree and the evaluation of relevant functions, and thus can use strongly targeted techniques accordingly. Thirdly, guide the rehabilitation therapy and reasonably adjust techniques in time. Based on the evaluation result of joint movement degree, one can make a correct scheme for manipulative therapy according to the joint function situation and causes for abnormal joint movement and choose a correct therapeutic method. Especially, this is extremely important for the selection of techniques for shoulder joint and other movable joints. Meanwhile, further inspect the curative effect of massage through dynamic evaluation.

\section{Massage can enrich the rehabilitation means and help to enhance the rehabilitation effect}

There are many methods to treat apoplectic hemiplegia by using massage, such as rolling, scraping, one-finger massaging, hitting and striking, pressing and rubbing. The massage is conducted from the far end to the near end. In particular, it is required to massaging the hand, shoulder and lower limb on the affected side, because this can help to improve the blood circulation, relieve swelling, alleviate pain and prevent the bedsore and phlebitis. In order to facilitate the functional recovery, the massage shall be conducted from the near end to the far end so as to promote the recovery of limb function on the affected side. Passive movements of all joints can be carried out after massage. Mainly carry out the movements of metacarpophalangeal joints and shoulder joints for the upper limb and the ankle joints for the lower limb. The combination with the modern rehabilitation training can not only enrich the means of rehabilitation but also yield twice the result with half the effort. As an effective, safe and convenient therapeutic means, massage is easily accepted by hemiplegic patients at both the early and recovery phases, so massage can help the recovery. Besides, massage can improve the curative effect and there are numerous clinical reports concerned ${ }^{[3-4]}$. The method of combining massage with rehabilitation can overcome their own weak points by learning from each other's strong points and bring out the best of each other to help patients to get well soon under the principle of not violating their respective theories.

\section{The kinesitherapy in modern rehabilitation can perfect its therapy system by using relevant content in massage method for reference.}

The kinesitherapy in modern rehabilitation unilaterally emphasizes the active or passive movements of patients and thus neglects the patients' emotion and subjective consciousness. However, the massage method can exactly make up for this deficiency ${ }^{[5]}$. Owing to the highly-intensive thinking activities, the massage training can effectively facilitate the conversion of 
"spirit, vital energy and blood", improve their functions and qualities, and thus help the body to generate the so-called "inner strength" by "leading the vital energy with thought and fill in strength with vital energy". The generation of such "inner strength" can effectively improve the curative effect of kinesitherapy in modern rehabilitation. For instance, a patient can do the actions such as the phoenix spreading the wings with the assistance of the rehabilitation therapist; the patient can crosswise spread the two arms toward two sides to expand his/her thorax; in this way, the upper-jiao Qi function can be stretched; this can help to loosen the chest, promote the circulation of Qi, calm the lever and smooth the lung; besides, the Qi function is also adjusted to lower the highly disobedient liver-Yang; therefore, this action can prevent the recurrence of stroke and stabilize blood pressure. In addition to the active or passive movements with the assistance of the rehabilitation therapist, the stroke-caused hemiplegic patients can also choose their own suitable methods for training. For instance, the gesture of a roc pressing a crop can enhance the shoulder's muscle strength and recover the normal functions of external rotation of shoulder joint. The stroke-caused hemiplegic patients' selection of their own suitable massage methods for training can effectively consolidate and enhance the rehabilitation effect and thus perfect the rehabilitation system.

In a word, the massage therapy is dominated by the passive movement and followed by the active training of modern rehabilitation therapist. The effective combination of massage therapy and modern rehabilitation method, which can help them to mutually learn each other's advantages and complement each other's advantages, is the perfect combination between traditional Chinese medical rehabilitation and modern rehabilitation, and is greatly beneficial to the stroke-caused hemiplegic patients' speedy recovery.

\section{References}

[1] Ji Shurong. Kinesitherapy technology, Huaxia Publishing House, 2009:342, 569.

[2] Zeng Qingyun, Wang Yongyuan and Ye Xiaoqin et al. Thinking and experience of fusion of Chinese and western medicine for treatment of stroke with massage, Modern Journal of Integrated Traditional Chinese and Western Medicine, 2011,20 (3): 325-326.

[3] Liu Yaoyuan. Massage therapy of sequela of apoplexy, Chinese Manipulation \& Qi Gong Therapy, 2007,23 (6): 34-35.

[4] Li Xiaojun. Massage therapy of sequela of apoplexy, Chinese Manipulation \& Qi Gong Therapy, 1998, 80 (3): 25-26.

[5] Wang Guocai. Science of massage manipulation, China Press of Traditional Chinese Medicine, 2007: 53. 